\title{
U(2) Invariant Four Dimensional Einstein Metrics
}

\section{Peter Petersen \&3 Shunhui Zhu}

\begin{abstract}
We construct explicitly most known examples of four dimensional Einstein metrics with $U(2)$ symmetry, in particular, we find many examples of compact 4-dimensional Einstein orbifolds with codimension 2 singularities, some of which have no manifold analogues.
\end{abstract}

1. Introduction. In this note we will discuss metrics of the form

$$
d r^{2}+\phi^{2}(r) \sigma_{1}^{2}+\psi^{2}(r)\left(\sigma_{2}^{2}+\sigma_{3}^{2}\right)
$$

where $\sigma_{i}$ is the standard orthonormal frame on $S^{3}$ satisfying $d \sigma_{i}=-2 \sigma_{i+1} \wedge \sigma_{i+2}$ (all indices are mod 3 ). Clearly $U(2)$ acts by isometries on the metrics, while the more general metrics

$$
d r^{2}+\phi_{1}^{2}(r) \sigma_{1}^{2}+\phi_{2}^{2}(r) \sigma_{2}^{2}+\phi_{3}^{2}(r) \sigma_{3}^{2}
$$

only have $S U(2)$ symmetry.

Metrics of this type were first studied in the physics literature. There was a fairly complete treatment in $[\mathrm{BB}]$, and indeed we do not come up with any new compact manifold examples. What we do is to show that there are also many interesting orbifolds of this type. Along the same line, we also observe that the examples in [Le] are of this type, thus very many interesting Kähler and non-Kähler metrics in dimension four with constant scalar curvature have this very elementary form and all their curvatures, including curvature operator can be computed explicitly.

All undefined notions and results mentioned in this note can be found in [Be]. Let us also point out that most of the computations in the actual metrics are done with Mathematica ${ }^{(}$, which we find quite helpful. 
2. Notations and computations. Denote by $\theta_{0}=d r, \theta_{1}=\phi \sigma_{1}, \theta_{2}=$ $\psi \sigma_{2}, \theta_{3}=\psi \sigma_{3}$ the standard orthonormal coframe on $I \times S^{3}$ with respect to the metric and $\partial / \partial r, \xi_{1}, \xi_{2}, \xi_{3}$ corresponding orthonormal frame. The projection $I \times S^{3} \longrightarrow I$ is a Riemannian submersion with homogeneous fibers, denoted by $S_{r}^{3}$. The metric on $S_{r}^{3}$ is $g_{r}=\theta_{1}^{2}+\theta_{2}^{2}+\theta_{3}^{2}$ and the shape operator (the second fundamental form) is

$$
S_{r}=\left(\begin{array}{ccc}
\dot{\phi} / \phi & 0 & 0 \\
0 & \dot{\psi} / \psi & 0 \\
0 & 0 & \dot{\psi} / \psi
\end{array}\right)
$$

All curvatures on $I \times S^{3}$ can now be computed using the formulae:

Tube formula (Evolution equation):

$$
\frac{d S_{r}}{d r}+S_{r}^{2}=\left(\begin{array}{ccc}
\ddot{\phi} / \phi & 0 & 0 \\
0 & \ddot{\psi} / \psi & 0 \\
0 & 0 & \ddot{\psi} / \psi
\end{array}\right)=-R\left(\cdot, \frac{\partial}{\partial r}\right) \frac{\partial}{\partial r} \quad \text { on } T S_{r}^{3}
$$

Gauss equation:

$$
\left\langle R\left(\xi_{i}, \xi_{j}\right) \xi_{k}, \xi_{\ell}\right\rangle=\left\langle\hat{R}\left(\xi_{i}, \xi_{j}\right) \xi_{k}, \xi_{\ell}\right\rangle+\left\langle S\left(\xi_{j}\right), \xi_{k}\right\rangle\left\langle S\left(\xi_{i}, \xi_{\ell}\right\rangle-\left\langle S\left(\xi_{i}\right), \xi_{k}\right\rangle\left\langle S\left(\xi_{j}\right), \xi_{\ell}\right\rangle\right.
$$

where $1 \leq i, j, k, \ell \leq 3, \hat{R}$ is the curvature tensor for $S_{r}^{3}$.

Codazzi-Mainardi:

$$
\begin{aligned}
\left\langle R\left(\xi_{i}, \xi_{j}\right) \xi_{k}, \frac{\partial}{\partial r}\right\rangle & =\left\langle\operatorname{Tor}_{S_{r}}\left(\xi_{i}, \xi_{j}\right), \xi_{k}\right\rangle \\
& =\left\langle D_{\xi_{i}} S\left(\xi_{j}\right)-D_{\xi_{j}} S\left(\xi_{i}\right)-S\left[\xi_{i}, \xi_{j}\right], \xi_{k}\right\rangle
\end{aligned}
$$

The curvature tensor of $\left(S_{r}^{3}, g_{r}\right)$ is easily seen to satisfy:

$$
\begin{aligned}
\hat{R}\left(\xi_{i}, \xi_{j}\right) \xi_{k} & =0 \text { if all indices are different } \\
\hat{R}\left(\xi_{i}, \xi_{i}\right) \xi_{i} & =\frac{\phi^{2}}{\psi^{4}} \xi_{i}, \quad i=2,3 \\
\hat{R}\left(\xi_{2}, \xi_{3}\right) \xi_{3} & =\frac{4 \psi^{2}-3 \phi^{2}}{\psi^{4}} \xi_{2}
\end{aligned}
$$

This, together with $S_{r}$, implies that $\left\langle R\left(\xi_{i}, \xi_{j}\right) \xi_{k}, \xi_{\ell}\right\rangle=0$ unless all indices are different or match up in pairs to give a sectional curvature and

$$
\begin{aligned}
S_{23}=\sec \left(\xi_{2}, \xi_{3}\right) & =\frac{4 \psi^{2}-3 \phi^{2}}{\psi^{4}}-\frac{\dot{\psi}^{2}}{\psi^{2}}, \\
S_{10}=\sec \left(\xi_{1}, \xi_{0}\right) & =-\frac{\ddot{\phi}}{\phi},
\end{aligned}
$$




$$
\begin{aligned}
S_{i 0}=\sec \left(\xi_{i}, \xi_{0}\right) & =-\frac{\ddot{\psi}}{\psi}, \quad i=2,3, \\
S_{1 i}=\sec \left(\xi_{1}, \xi_{i}\right) & =\frac{\phi^{2}}{\psi^{4}}-\frac{\dot{\phi} \dot{\psi}}{\phi \psi}, \quad i=2,3, \\
\left\langle R\left(\xi_{1}, \xi_{2}\right) \xi_{3}, \xi_{0}\right\rangle & =M:=\frac{\dot{\phi} \psi-\phi \dot{\psi}}{\psi^{3}} .
\end{aligned}
$$

As we are in dimension 4 , we should write the curvature operator up in a matrix with respect to a selfdual (anti-selfdual) basis on $\bigwedge^{2} T M=\Lambda_{+}^{2} \oplus \bigwedge_{-}^{2}$. Our orientation is $\xi_{0}, \xi_{1}, \xi_{2}, \xi_{3}$, consequently

$$
\xi_{0} \wedge \xi_{1} \pm \xi_{2} \wedge \xi_{3}, \quad \xi_{0} \wedge \xi_{2} \pm \xi_{3} \wedge \xi_{1}, \quad \xi_{0} \wedge \xi_{3} \pm \xi_{1} \wedge \xi_{2}
$$

span $\bigwedge_{ \pm}^{2} T M$. Normalizing these vectors by $1 / \sqrt{2}$ yields an orthonormal basis for $\bigwedge_{ \pm}^{2} T M$ and in this basis we can write the curvature operator as

$$
R=\left(\begin{array}{cc}
A & B \\
B^{*} & D
\end{array}\right)
$$

where all matrices are diagonal matrices of size 3 with the diagonal elements:

$$
\begin{aligned}
& B=\frac{1}{2} \operatorname{diag}\left(-S_{01}+S_{23},-S_{02}+S_{13},-S_{03}+S_{12}\right) \\
& A=\frac{1}{2} \operatorname{diag}\left(S_{01}+S_{23}-4 M, S_{02}+S_{13}+2 M, S_{03}+S_{12}+2 M\right) \\
& D=\frac{1}{2} \operatorname{diag}\left(S_{01}+S_{23}+4 M, S_{02}+S_{13}-2 M, S_{03}+S_{12}-2 M\right)
\end{aligned}
$$

Notice that in all of these matrices the last two diagonal elements are identical. This implies that $W^{+}=A-\frac{1}{12}$ scal, $W^{-}=D-\frac{1}{12}$ scal only have two eigenvalues. Hence these metrics are conformal to Kähler metrics as long as $\left|W^{+}\right| \neq 0$ (or $\left|W^{-}\right| \neq 0$ ) by a result of Derdzinski ([De]).

There is a natural almost complex structure defined by

$$
J\left(\xi_{0}\right)=\xi_{1}, \quad J\left(\xi_{1}\right)=-\xi_{0}, \quad J\left(\xi_{2}\right)=\xi_{3}, \quad J\left(\xi_{3}\right)=-\xi_{2} .
$$

It is easy to check directly that the torsion vanishes, thus the structure is integrable, and our metrics are in fact Hermitian. The structure $J$ is, however, only parallel when $\phi=\psi \dot{\psi}$ in which case the metric becomes Kählerian. Conversely we can prove that any (complete) Ricci flat Kähler metric of this form must satisfy $\phi=\psi \dot{\psi}$. This is because our metric is Einstein if and only if $B=0$ (seen by a simple algebraic calculation of $[\mathrm{AS}]$ ). So if the metric is Ricci flat we get that:

$$
\begin{aligned}
W^{ \pm} & =\operatorname{diag}\left(-\frac{\ddot{\phi}}{\phi} \mp 2 \frac{\dot{\phi} \psi-\phi \dot{\psi}}{\psi^{3}},-\frac{\ddot{\psi}}{\psi} \pm \frac{\dot{\phi} \psi-\phi \dot{\psi}}{\psi^{3}},-\frac{\ddot{\psi}}{\psi} \pm \frac{\dot{\phi} \psi-\phi \dot{\psi}}{\psi^{3}}\right) \\
& =\operatorname{diag}\left(2\left(\frac{\ddot{\psi}}{\psi} \mp \frac{\dot{\phi} \psi-\phi \dot{\psi}}{\psi^{3}}\right),-\frac{\ddot{\psi}}{\psi} \pm \frac{\dot{\phi} \psi-\phi \dot{\psi}}{\psi^{3}},-\frac{\ddot{\psi}}{\psi} \pm \frac{\dot{\phi} \psi-\phi \dot{\psi}}{\psi^{3}}\right)
\end{aligned}
$$




$$
=\operatorname{diag}\left(\frac{2}{\psi} \frac{d}{d t}\left(\dot{\psi} \mp \frac{\phi}{\psi}\right), \frac{1}{\psi} \frac{d}{d t}\left(-\dot{\psi} \pm \frac{\phi}{\psi}\right), \frac{1}{\psi} \frac{d}{d t}\left(-\dot{\psi} \pm \frac{\phi}{\psi}\right)\right) .
$$

Now, if $M$ is Kähler and has scal $=0$, then $\left|W^{+}\right|=0$ by [De]. Since an orientation change will interchange $W^{+}$and $W^{-}$, we must check that both are non-zero to eliminate the possibility of the metric being Kählerian. Our calculations of $W^{ \pm}$show, however, that $\left|W^{ \pm}\right|=0$ only when $\dot{\psi} \mp \phi / \psi=$ constant $=k$. To have a complete metric with nonzero $\dot{\psi}$, we will always have to assume $\phi(0)=0$ and either $\psi(0)>0, \dot{\psi}(0)=0$ or $\psi(0)=0, \dot{\psi}(0)=1$. In either case, this implies that $k=0$ and hence $\phi= \pm \psi \dot{\psi}$.

The condition $B=0$ also supplies us with the equations we need to solve in order to get Einstein metrics. Explicitly we get two equations:

$$
\begin{aligned}
& S_{01}=S_{23}:-\frac{\ddot{\phi}}{\phi}=\frac{4 \psi^{2}-3 \phi^{2}}{\psi^{4}}-\frac{\dot{\psi}^{2}}{\psi^{2}}, \\
& S_{02}=S_{13}, \text { or } \quad S_{03}=S_{12}:-\frac{\ddot{\psi}}{\psi}=\frac{\phi^{2}}{\psi^{4}}-\frac{\dot{\phi} \dot{\psi}}{\phi \psi} .
\end{aligned}
$$

The Einstein constant is $\operatorname{Ric}\left(\xi_{0}, \xi_{0}\right)=-\ddot{\phi} / \phi-2 \ddot{\psi} / \psi=\alpha$.

To solve these equations we assume $\phi=F(\psi) \dot{\psi}$, and $\dot{\psi}^{2}=G(\psi)$, which is valid as long as $\dot{\psi} \neq 0$. And the $\dot{\psi}=0$ case is not too interesting besides not yielding an Einstein metric. With these relations we get: $\dot{\phi}=F^{\prime} G+\frac{1}{2} F G^{\prime}$, $\ddot{\psi}=G^{\prime} / 2$ and $\ddot{\phi} / \phi=-2 \ddot{\psi} / \psi-\alpha=-G^{\prime} / \psi-\alpha$. Inserting these into (1) and (2) yields:

$$
\begin{aligned}
G^{\prime}-\left(\frac{3 F}{\psi^{3}}+\frac{1}{\psi}\right) G & =\frac{4}{\psi}-\alpha \psi, \\
F^{\prime} & =\frac{F^{3}}{\psi^{3}} .
\end{aligned}
$$

(4) immediately implies $F^{2}=\psi^{2} /\left(1+c \psi^{2}\right)$ for some constant $c$. When $c=0$ we are therefore in the Kähler case. Substituting this into (3) yields:

$$
G^{\prime}+\left(\frac{4}{\psi}-\frac{3 c \psi}{1+c \psi^{2}}\right) G=\frac{4}{\psi}-\alpha \psi
$$

which has the solution:

$$
\begin{aligned}
& c=0 \quad: \quad \dot{\psi}^{2}=G(\psi)=1-\frac{\alpha}{6} \psi^{2}+k \psi^{-4} \\
& c \neq 0 \quad: \quad \dot{\psi}^{2}=G(\psi)=\frac{u^{2}}{\psi^{4}}\left(\frac{4}{c^{2}}\left(u^{2}+1\right)-\frac{\alpha}{c^{3}}\left(\frac{1}{3} u^{4}-2 u^{2}-1\right)+k u\right),
\end{aligned}
$$

where $u^{2}=1+c \psi^{2}$ and $k$ is some integration constant.

Suppose that $\phi=\psi \dot{\psi}$ (Kähler case) we can even solve the scal $=0$ equation using that we already know $\dot{\psi}^{2}=1+k \psi^{-4}$ is an integral. Namely,

$$
\text { scal }=-G^{\prime \prime}-\frac{7}{\psi} G^{\prime}-\frac{8}{\psi^{2}} G+\frac{8}{\psi^{2}}=0
$$


has the solution $G=1$ and the general solution to the Euler equation is $A \psi^{-2}+$ $B \psi^{-4}$, yielding $\dot{\psi}^{2}=G(\psi)=1+A \psi^{-2}+B \psi^{-4}$ as the solutions with scal $=0$ and $J$ as Kähler structure.

We are now ready to find all the described metrics. What we need to worry about is whether we get complete smooth metrics. First, let us normalize so that $r \geq 0$. The metric will now be smooth at $r=0$ if one of the following holds

$$
\begin{aligned}
& \phi(0)=\psi(0)=0, \dot{\phi}(0)=\dot{\psi}(0)=1, \phi^{(\text {even })}(0)=\psi^{(\text {even })}(0)=0 \\
& \psi(0)>0, \psi^{(\text {odd })}(0)=0, \phi(0)=\phi^{(\text {even })}=0, \dot{\phi}(0)=\text { integer } \geq 1 .
\end{aligned}
$$

In the case when $\dot{\phi}(0)=p \geq 2$, the smooth metric is on $I \times\left(S^{3} / Z_{p}\right)$ rather than $I \times S^{3}$. When $\dot{\phi}(0)=p / q$ and $\dot{\psi}(0)=0$ then we get an orbifold metric on $I \times\left(S^{3} / Z_{p}\right)$, where the singular set at $r=0$ is $S^{2}$ with a cone metric with cone angle $2 \pi / q$.

For compact examples we need $\phi(b)=0$ for some $b>0$ and all the above smooth criteria while for a complete metric we must check that $\psi, \phi>0$ for all $r>0$ and exist for all time.

Since $\phi, \psi$ solve the above equations and $\phi=F(\psi) \dot{\psi}$ it is easy to see that conditions on the higher derivatives of $\phi, \psi$ are automatic. Furthermore $\phi(a)=0$ if $\psi(a)$ or $\dot{\psi}(a)=0$. So everything depends on analyzing $\psi$ from the equations:

$$
\begin{aligned}
& c=0 \quad: \quad \dot{\psi}^{2}=1-\frac{\alpha}{6} \psi^{2}+k \psi^{-4}, \\
& c \neq 0 \quad: \quad \dot{\psi}^{2}=\frac{u^{2}}{\psi^{4}}\left(\frac{4}{c^{2}}\left(u^{2}+1\right)-\frac{\alpha}{c^{3}}\left(\frac{1}{3} u^{4}-2 u^{2}-1\right)+k u\right) .
\end{aligned}
$$

3. The actual metrics. We now solve the boundary value problem above in all cases. We will first consider the three different cases according to the sign of the constant $c$, i.e., $c=0,1,-1$. In each of these cases, we will subdivide acoording to the two types of boundary values, i.e., $\psi(0)=0$ or $\psi(0)>0$. The sign of the Einstein constant will be further considered in each of these cases. We will give a summarizing table at the end of the section.

In what follows, we will denote by $E_{k}$ the complex line bundle over $C P^{1}$ with Euler number $k$.

Case $c=0$ (Kählerian case). The equation is

$$
\left\{\begin{array}{l}
\phi=\psi \dot{\psi} \\
\dot{\psi}^{2}=1-\frac{\alpha}{6} \psi^{2}+k \psi^{-4}
\end{array}\right.
$$

We consider the two kinds of initial conditions: $\psi(0)=0$ and $\psi(0)>0$.

(1) $\psi(0)=0$. Smoothness requires $\dot{\psi}(0)=1$, thus forces $k=0$.

The equation for $\psi$ becomes

$$
\left\{\begin{array}{l}
\dot{\psi}^{2}=1-\frac{\alpha}{6} \psi^{2} \\
\psi(0)=0 .
\end{array}\right.
$$


By scaling (since $c=0$, scaling is OK), we can easily solve the three cases to get

(a) $\alpha=6$ : Canonical metric on $C P^{2}$.

(b) $\alpha=0$ : Canonical metric on $R^{4}$.

(c) $\alpha=-6: C H^{2}$, i.e., the Kähler Einstein metric on $R^{4}$ with Ricc $=-6$ (the counterpart to $C P^{2}$, not the hyperbolic metric).

(2) $\psi(0)>0$.

Again we can scale the metric to get $\psi(0)=1$. Smoothness at $t=0$ requires $\dot{\psi}(0)=0$. Hence $k=\alpha / 6-1$. The equation now becomes

$$
\left\{\begin{array}{l}
\dot{\psi}^{2}=1-\frac{\alpha}{6} \psi^{2}+\left(\frac{\alpha}{6}-1\right) \psi^{-4} \\
\psi(0)=1
\end{array}\right.
$$

which can be factorized to be

$$
\dot{\psi}^{2}=\frac{\alpha}{6} \psi^{-4}\left(1-\psi^{2}\right)\left(\psi^{4}+\left(1-\frac{6}{\alpha}\right) \psi^{2}+\left(1-\frac{6}{\alpha}\right)\right) .
$$

Note the initial condition for $\phi$ is $\phi(0)=0$ and $\dot{\phi}(0)=2-\alpha / 2$.

When $\alpha \leq 0$, the right hand side of this equation has no zero except at $t=0$. Thus the solution exists for all time. We therefore get complete metrics in this case.

(a) $\alpha=0$ : Eguchi-Hanson metric $([\mathrm{EH}])$ on $E_{2}=T S^{2}$.

(b) $\alpha<0$ : $(\alpha=2(2-\chi)$ for $\chi>2)$. Kähler Einstein metric on $E_{\chi}$.

(c) $\alpha>0$ : This case needs further analysis. Without loss of generality, we assume $\dot{\psi}_{\text {.. }}>0$ (otherwise we can just reparametrize $t$ ); thus, $\alpha \leq 4$ since $\ddot{\psi}(0)=2-\alpha / 2$. The solution will exist in an interval $\left[0, t_{0}\right]$ such that $\psi\left(t_{0}\right)$ is a real zero in $(1,+\infty)$ of the right hand side of the equation, i.e.

$$
\psi\left(t_{0}\right)=\sqrt{\frac{\frac{6}{\alpha}-1+\sqrt{3\left(1+\frac{2}{\alpha}\right)\left(\frac{6}{\alpha}-1\right)}}{2}} .
$$

Note for $\alpha \in(0,4], \psi\left(t_{0}\right)$ is always bigger than 1 . Thus the solution always exists in a finite interval (this of course also follows from Myers' Theorem). Note that $\psi$ satisfies the smoothness condition at 0 and $t_{0}$. We will now consider the function $\phi$, which satisfies

$$
\left\{\begin{array}{l}
\phi(0)=0, \quad \dot{\phi}(0)=2-\frac{\alpha}{2} \\
\phi\left(t_{0}\right)=0, \quad \dot{\phi}\left(t_{0}\right)=1-\frac{\alpha}{3} \psi^{2}\left(t_{0}\right)-\left(\frac{\alpha}{6}-1\right) \psi^{-4}\left(t_{0}\right) .
\end{array}\right.
$$


To get an orbifold metric we need that $\dot{\phi}(0), \dot{\phi}\left(t_{0}\right)$ both be rational numbers, i.e.,

$$
\left\{\begin{array}{l}
2-\frac{\alpha}{2}=\frac{\ell}{m} \\
1-\frac{\alpha}{3} \psi^{2}\left(t_{0}\right)-\left(\frac{\alpha}{6}-1\right) \psi^{-4}\left(t_{0}\right)=-\frac{\ell}{n},
\end{array}\right.
$$

with $\psi\left(t_{0}\right)$ given in terms of $\alpha$ as in (5).

Any solution to this system of algebraic equations give rise to a complex Kähler Einstein orbifold with positive Ricci curvature whose underlying space is $E_{\ell} \cup \bar{E}_{\ell}$ and the orbifold singularity contains two $C P^{1}$ components with isotropy groups $Z_{m}$ and $Z_{n}$.

There are infinitely many solutions to the equations above. The smallest (in terms of $\ell, m, n$ ) is $\ell=18, m=14, n=21$, (thus $\alpha=\frac{10}{7}$, $\left.\psi\left(t_{0}\right)=2\right)$.

(3) Scalar flat metrics.

We now consider briefly scalar flat Kähler metrics.

As computed previously, the equation is

$$
\left\{\begin{array}{cl}
\phi & =\psi \dot{\psi} \\
\dot{\psi}^{2} & =1+A \psi^{-2}+B \psi^{-4}
\end{array}\right.
$$

Note also $\dot{\phi}=1-B \psi^{-5}$.

The case $\psi(0)=0$ yields the flat Euclidean metric. In the case when $\psi(0)>0$, without loss of generality, we assume $\psi(0)=1$, the smoothness at $t=0$ gives

$$
1+A+B=0
$$

and therefore:

$$
\dot{\psi}^{2}=\frac{(\psi+1)(\psi-1)\left(\psi^{2}-B\right)}{\psi^{4}} .
$$

We will discuss two cases.

(a) If $B>0$ and $B \neq 1$ : In this case we get a solution $\psi$ that exists in $\left[0, t_{0}\right]$ with $\psi\left(t_{0}\right)=\sqrt{B}$. Thus

$$
\dot{\phi}(0)=1-B, \quad \dot{\phi}\left(t_{0}\right)=1-B^{-3 / 2}
$$

We therefore will not get any manifold examples. One can of course get many orbifold metrics in this case, but we will not discuss this further.

(b) If $B \leq 0$ or $B=1$ : In both cases, $\psi$ exists for all time. Note $B=1$ implies $\dot{\phi}(0)=0$, which does not give smooth metrics. The case $B=-n$ (for $n$ a nonnegative integer) gives a complete scalar flat 
Kähler metric on $E_{n+1} . \quad n=0$ is the Burns metric on the blowup of $C^{2}$ at the origin. $n=1$ is the Eguchi-Hanson metric. For $n \geq 2$, these are complete asymptotically flat manifolds due to LeBrun ([Le]), who showed that they all have negative mass, thus giving counter-examples to the generalized positive action conjecture of Hawking and Pope. 
Case $c=1$ (nonKähler).

The equation is now

$$
\left\{\begin{array}{l}
\phi=\psi \dot{\psi} / u \\
\dot{\psi}^{2}=u^{2} \psi^{-4}\left(4\left(u^{2}+1\right)-\alpha\left(\frac{u^{4}}{3}-2 u^{2}-1\right)+k u\right),
\end{array}\right.
$$

where $u=\sqrt{1+\psi^{2}}$.

(1) $\psi(0)=0$. The regularity at $t=0$ gives $k=-8(\alpha / 3+1)$.

An easy calculation shows $\dot{\psi}(0)=1$. Thus $\psi$ is smooth at the origin and it satișies,

$$
\left\{\begin{array}{l}
\dot{\psi}^{2}=u^{2} \psi^{-4}\left(4\left(u^{2}+1\right)-\alpha\left(u^{4} / 3-2 u^{2}-1\right)-8(\alpha / 3+1) u\right) \\
\psi(0)=0
\end{array}\right.
$$

This equation can be further factorized to give

$$
\dot{\psi}^{2}=-\frac{\alpha}{3} u^{2}(u-1)^{2} \psi^{-4}\left(u^{2}+2 u-(3+12 / \alpha)\right)
$$

Considering the order of both sides, we see that the solution exists for the whole half interval $[0, \infty)$ unless there is a zero in $(1, \infty)$ for the last quadratic term. This zero is given by

$$
u=\sqrt{4+12 / \alpha}-1
$$

Thus when $\alpha \leq 0$, the solution to (6) exists for all time. Note that for all $\alpha$, we always have $\phi(0)=0, \dot{\phi}(0)=1$. Therefore both $\phi$ and $\psi$ satisfy the smoothness condition at $t=0$.

(a) $\alpha=0$ : A Ricci flat nonKähler metric on $R^{4}$.

(b) $\alpha<0$ : A countinous family of Einstein metrics on $R^{4}$ containing the hyperbolic metric (when $\alpha=-3$ ).

(c) $\alpha>0$ : Just as before, we have to analyse this case further.

Since $\psi$ is increasing, the equation can be written as

$$
\dot{\psi}=\sqrt{\frac{\alpha}{3}} u(u-1) \psi^{-2} \sqrt{-u^{2}-2 u+(3+12 / \alpha)}
$$

The solution exists in $\left[0, t_{0}\right]$, such that

$$
u\left(t_{0}\right)=2 \sqrt{1+3 / \alpha}-1
$$

To see whether we get an orbifold metric (compact from the existence of $t_{0}$ or Myer's theorem), we need to look at $\phi$ at $t_{0}$. Straightforward computation gives

$$
\phi\left(t_{0}\right)=0, \quad \dot{\phi}\left(t_{0}\right)=\frac{2 \alpha(1-\sqrt{1+3 / \alpha})}{3},
$$

where we used (7) for $u\left(t_{0}\right)$. 
When $\alpha=3 m^{2} / 4 n(n-m)$ with $n>m$, we have $\dot{\phi}\left(t_{0}\right)=-m / n$. This gives rise to a compact nonKähler Einstein orbifold whose underlying space is the one point compactification of $E_{m}$. When $m \neq 1$, the orbifold singularity has one $C P^{1}$ component with isotropy $Z_{n}$ and a point singularity with isotropy $Z_{m}$. When $m=1$, the singularity consists only the $C P^{1}$ component. We point out there is no manifold analogue of this metric, it is strictly an orbifold phenomenon.

(2) $\psi(0)>0$.

This case is more complicated, so we will take a different approach. Denote

$$
P(u)=4\left(u^{2}+1\right)-\alpha\left(u^{4} / 3-2 u^{2}-1\right)+k u .
$$

At a point where $P(u)=0$, we have

$$
\dot{\phi}(u)=\frac{P^{\prime}(u)}{2\left(u^{2}-1\right)} .
$$

If $u(0)>1$ is a root of $P(u)$, then we can write $k$ in terms of $u(0)$ and $\alpha$, and direct computation shows

$$
\dot{\phi}(0)=\frac{4-\alpha \psi^{2}(0)}{2 \sqrt{1+\psi^{2}(0)}} .
$$

On the other hand, if $P(u)$ has two distinct solutions $u\left(t_{0}\right)>u(0)>1$, then solving for $\alpha, k$ gives (we only give $\alpha$ here)

$$
\alpha=\frac{12\left(u(0) u\left(t_{0}\right)-1\right)}{u(0) u^{3}\left(t_{0}\right)+u^{2}(0) u^{2}\left(t_{0}\right)+u^{3}(0) u\left(t_{0}\right)-6 u(0) u\left(t_{0}\right)+3}
$$

Note that if $u\left(t_{0}\right)>u(0)>1$ we have $\alpha>0$. Thus when $\alpha \leq 0, P(u)$ can have only one solution in $(1, \infty)$. The metric in this case is therefore complete.

(a) $\alpha=0$ : We have $\dot{\phi}(0)=2 / \sqrt{1+\psi^{2}(0)}$. Thus the only manifold solution is when $\psi(0)=\sqrt{3}$, i.e., $\dot{\phi}(0)=1$. This is a complete nonKähler Ricci flat metric on $E_{1}=\tau\left(C P^{1}\right)$.

(b) $\alpha<0$ : For any complex line bundle $E$ over $C P^{1}$, there is a family of Einstein metrics with negative Ricci curvature (e.g., when $\alpha=-1, \psi(0)=$ $\sqrt{2 \chi^{2}-4+2 \chi \sqrt{\chi^{2}-3}}$ for $\chi \geq 3$ and $\alpha=-\frac{1}{4}, \psi(0)=2 \sqrt{2}$ on $\tau\left(C P^{1}\right)$.)

(c) $\alpha>0$ : since $\dot{\phi}$ as given by (8) is a rational function of $u(0), u\left(t_{0}\right)$, we can choose any rational number $u\left(t_{0}\right)>u(0)>1$. This always gives an orbifold metric. The underlying space is $E_{\ell} \cup \bar{E}_{\ell}$, with the singular set consisting of two copies of $C P^{1}$. (For example, when $u(0)=2, u\left(t_{0}\right)=3$, we get $\alpha=\frac{20}{27}, \dot{\phi}(0)=\frac{4}{9}$ and $\dot{\phi}\left(t_{0}\right)=-\frac{26}{81}$. Thus the line bundle is $E_{52}$, and the sinularities have isotropy groups $Z_{117}$ and $Z_{162}$.) 
Case $c=-1$ (nonKähler).

The equation is now:

$$
\left\{\begin{array}{l}
\phi=\psi \dot{\psi} / u \\
\dot{\psi}^{2}=u^{2} \psi^{-4}\left(4\left(u^{2}+1\right)+\alpha\left(\frac{u^{4}}{3}-2 u^{2}-1\right)+k u\right)
\end{array}\right.
$$

where $u=\sqrt{1-\psi^{2}}$.

(1) $\psi(0)=0$. The regularity at $t=0$ gives $k=8(\alpha / 3-1)$.

An easy computation shows

$$
\dot{\psi}(0)=1 \text { and } \phi(0)=0, \dot{\phi}(0)=1 \text {. }
$$

Thus both $\phi, \psi$ satisfy the smoothness condition at $t=0$.

The equation for $\psi$ is now

$$
\dot{\psi}=\sqrt{\frac{\alpha}{3}} u(1-u) \psi^{-2}\left(u^{2}+2 u+3\left(\frac{12}{\alpha}-3\right)\right) .
$$

The right hand side has two nonnegative zeros given by

$$
u=0, \quad u=2 \sqrt{1-\frac{3}{\alpha}}-1
$$

with order $\frac{1}{2}$. Thus the solution exists for a finite interval $\left[0, t_{0}\right]$, such that

$$
u\left(t_{0}\right)=0, \text { or } u\left(t_{0}\right)=2 \sqrt{1-\frac{3}{\alpha}}-1 \text { when it is in }(0,1)
$$

If $u\left(t_{0}\right)=0$, i.e., $\alpha<4$, computation gives

$$
\phi\left(t_{0}\right)=\sqrt{4-\alpha}, \quad \dot{\phi}\left(t_{0}\right)=\frac{4}{3}(3-\alpha)
$$

To get a compact orbifold metric, we need either $\phi\left(t_{0}\right)>0, \dot{\phi}\left(t_{0}\right)=0$ or $\phi\left(t_{0}\right)=0, \dot{\phi}\left(t_{0}\right)$ a negative rational number. This gives two possibilities:

(a) $\alpha=3$, which gives the standard $S^{4}(1)$ and $R P^{4}$;

(b) $\alpha=4,\left(\dot{\phi}\left(t_{0}\right)=-\frac{4}{3}\right)$, an Einstein orbifold whose underlying space is the one point compactification of $E_{4}$. The singular set has two components, a point with isotropy group $Z_{4}$ and a $C P^{1}$ with isotropy group $Z_{3}$.

If $u\left(t_{0}\right)=2 \sqrt{1-3 / \alpha}-1 \in(0,1)$, i.e., $\alpha>4$, we then have

$$
\left\{\begin{aligned}
& \phi\left(t_{0}\right)=0 \\
& \dot{\phi}\left(t_{0}\right)=\frac{1}{3} \psi\left(t_{0}\right)^{-4}\left[(24-8 \alpha)+(4 \alpha-12) \psi^{2}\left(t_{0}\right)\right. \\
&\left.+\left(\alpha \psi^{4}\left(t_{0}\right)+8 \alpha-24\right) \sqrt{1-\psi^{2}\left(t_{0}\right)}\right]
\end{aligned}\right.
$$


With the condition $u\left(t_{0}\right)=2 \sqrt{1-3 / \alpha}-1$, this formula for $\dot{\phi}\left(t_{0}\right)$ becomes very complicated. We will just point out that there are infinitely many $\alpha$ 's that will make $\dot{\phi}\left(t_{0}\right)$ a negative rational number. For example, when $\alpha=3\left(1+m^{2}\right)^{2} /\left(1+2 m^{2}\right)$, one computes

$$
\dot{\phi}\left(t_{0}\right)=\frac{2 m\left(1+m^{2}\right)\left(1+4 m+6 m^{2}+2 m^{3}+m^{4}-2 m^{5}\right)}{(1+m)^{4}\left(1+2 m^{2}\right)}
$$

which is negative rational when $m \geq 3(m=3$ gives $\alpha=300 / 19$ and $\left.\dot{\phi}\left(t_{0}\right)=-1065 / 304\right)$.

To summarize this case,

(a) $\alpha=0$ : No solution.

(b) $\alpha<0$ : No solution.

(c) $\alpha>0$ : There is an isolated solution when $\alpha=3$ which gives $S^{4}(1)$ and $R P^{4}$, and there is a rational family of Einstein orbifolds when $\alpha \geq 4$ whose underlyine space is an one point compactification of a comples line bundle over $C P^{1}$ with two singular components consisting of a point and a $C P^{1}$.

(2) $\psi(0)>0$.

Denote $P(u)=4\left(u^{2}+1\right)+\alpha\left(u^{4} / 3-2 u^{2}-1\right)+k u$. At the place where $P(u)=0$, it is easy to compute

$$
\dot{\phi}(u)=\frac{P^{\prime}(u)}{2\left(u^{2}-1\right)} .
$$

If $0<u(0)<1$ is a root of $P(u)$, then solving for $k$ in terms of $u(0)$ and $\alpha$, and using the above formula, we get

$$
\dot{\phi}(0)=\frac{4-\alpha \psi^{2}(0)}{2 \sqrt{1-\psi^{2}(0)}} .
$$

Note $G(u)=u^{2} P(u) /\left(1-u^{2}\right)^{2}$ always has $u=0$ as a root. Thus, $\psi$ will achieve 1 in finite time (unless 0 is a zero of $\mathrm{G}$ of order at least 4 , but that implies $\alpha=4$ and $k=0$, in which case no solution exsits). Thus the solution of $\psi$ will always exist only for a finite time.

If $u(0), u\left(t_{0}\right)$ are zeros of $P(u)$ such that $0<u\left(t_{0}\right)<u(0)<1$, we obtain

$$
\alpha=\frac{12\left(1-u(0) u\left(t_{0}\right)\right)}{u(0) u^{3}\left(t_{0}\right)+u^{2}(0) u^{2}\left(t_{0}\right)+u^{3}(0) u\left(t_{0}\right)-6 u(0) u\left(t_{0}\right)+3} .
$$

A simple calculation shows this is always positive in $(0,1) \times(0,1)$. Thus

(a) $\alpha=0$ : No solution.

(b) $\alpha<0$ : No solution. 
(c) $\alpha>0$ : We consider first the case when $P(u)$ has only one zero $u(0) \in$ $(0,1)$ and $t_{0}$ is such that $u\left(t_{0}\right)=0$, then since $\phi\left(t_{0}\right)=\sqrt{P(0)} \neq 0$, thus smoothness requires

$$
\dot{\phi}\left(t_{0}\right)=\frac{4\left(u^{2}(0)+1\right)+\alpha\left(u^{4}(0) / 3-2 u^{2}(0)-1\right)}{2 u(0)}=0 .
$$

This gives $\alpha$ in terms of $u(0)$, substituting into (9) gives

$$
\dot{\phi}(0)=\frac{4 u(0)\left(3+u^{2}(0)\right)}{3+6 u^{2}(0)-u^{4}(0)}
$$

The only time the above can be a positive integer for some $u(0) \in$ $(0,1)$ is when it is 1 . In this case $\dot{\phi}(0)=1, u(0) \approx 0.2817$, and $\alpha \approx$ 3.7328. This is the well-known Page's metric ([Pa]) on $C P^{2} \sharp \overline{C P^{2}}$. The case when $P(u)$ has two distinct solutions in $(0,1)$, we can take exactly the same approach as in the corresponding case when $c=1$ and obtain infinitely many orbifold metrics of the type $E_{m} \cup \overline{E_{m}}$. For example, when $u(0)=\frac{2}{3}, u\left(t_{0}\right)=\frac{1}{3}$, we have $\alpha=\frac{756}{149}, k=\frac{776}{149}$ and $\dot{\phi}(0)=\frac{132}{149}, \dot{\phi}\left(t_{0}\right)=-114 / 149$. Thus: the orbifold is the double of the complex line bundle $E_{2508}$ over $C P^{1}$ with $\chi=2508$ and two singular $C P^{1}$ 's with isotropy groups $Z_{2831}$ and $Z_{2508}$.

We summarize the discussion above in the following table. 


\begin{tabular}{|c|c|c|c|}
\hline \multirow{6}{*}{$\begin{array}{c}c=0 \\
\text { (Kähler) }\end{array}$} & \multirow{3}{*}{$\psi(0)=0$} & $\alpha=0$ & $R^{4}$ (Euclidean) \\
\hline & & $\alpha>0$ & $C P^{2}$ (Fubini-Study) \\
\hline & & $\alpha<0$ & $C H^{2}$ \\
\hline & \multirow{3}{*}{$\psi(0)>0$} & $\alpha=0$ & $T S^{2}$ (Eguchi-Hansen) \\
\hline & & $\alpha>0$ & (orbifolds) \\
\hline & & $\alpha<0$ & $E_{\chi}$ for $\chi \geq 3$ \\
\hline \multirow{6}{*}{ (nonKähler) } & \multirow{3}{*}{$\psi(0)=0$} & $\alpha=0$ & $R^{4}$ \\
\hline & & $\alpha>0$ & (orbifolds) \\
\hline & & $\alpha<0$ & $\begin{array}{l}R^{4} \text { (a continuous family containing } \\
\text { the hyperbolic metric) }\end{array}$ \\
\hline & \multirow{3}{*}{$\psi(0)>0$} & $\alpha=0$ & $\tau\left(C P^{1}\right)$ \\
\hline & & $\alpha>0$ & (orbifolds) \\
\hline & & $\alpha<0$ & $E_{\chi}$ for any $\chi$ \\
\hline
\end{tabular}




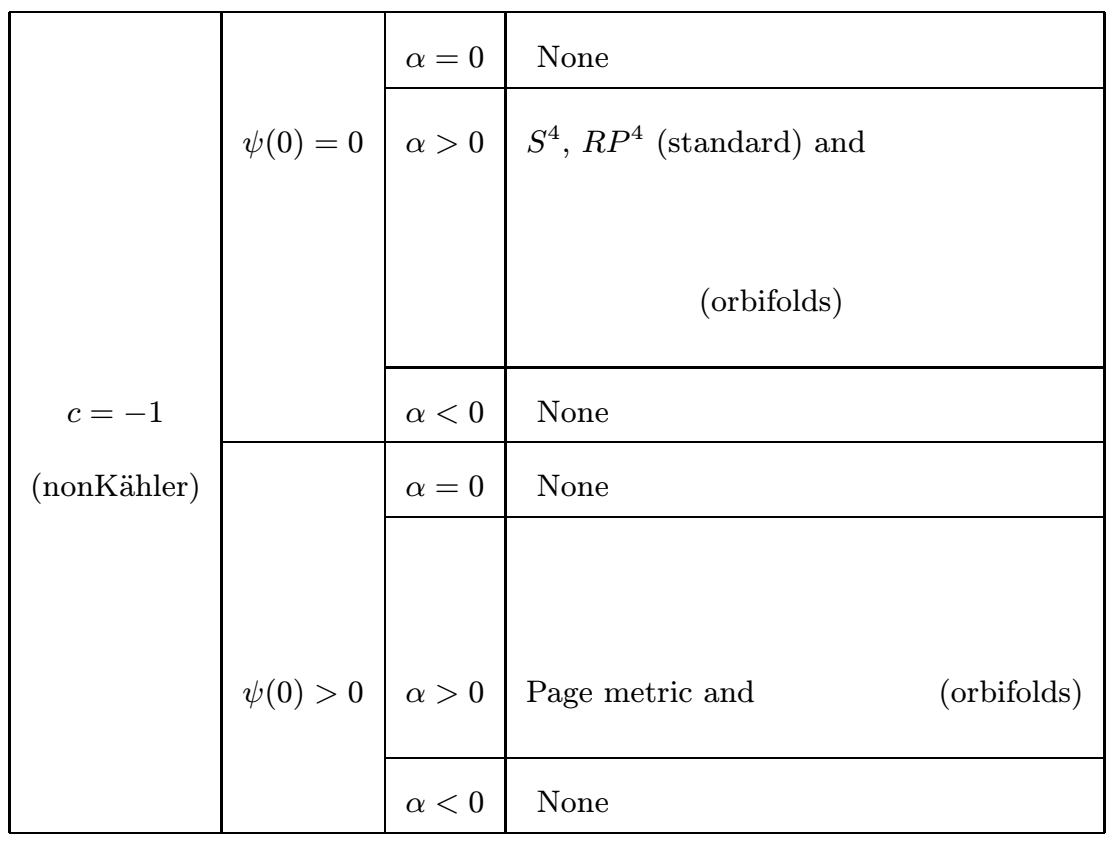

\section{REFERENCES}

[AS] W. Ambrose and I. M. Singer, A theorem on holonomy, Trans. Amer. Math. Soc., 79 (1953), 428-443.

[BB] L. Bérard Bergery, Sur de nouvelles variétés riemanniennes d'Einstein, Publications de l'Institut E. Cartan, 4 (1982), 1-60.

[Be] A. Besse, Einstein manifolds, Springer-Verlag, 1987.

[De] A. Derdzinski, Self-dual Käler manifolds and Einstein manifolds of dimension four, Compositio. Math., 49 (1983), 405-433.

[EH] T. Eguchi, A. J. Hanson, Asymptotically flat self-dual solutions to euclidean gravity, Phys. Lett., 237 (1978), 249-251.

[Le] C. LeBrun, Counter-examples to the generalized positive action conjecture, Comm. Math. Physics, 118 (1988), 591-596.

[Pa] D. PAGE, A compact rotating gravitational instanton, Phys. Lett., 79B (1979), 235-238.

[Pe] H. Pedersen, Einstein metrics, spinning-top motions, and monopoles, Math Ann., 274 (1986), 35-59.

Research by both authors was supported in part by a grant from the National Science Foundation. The first author was also supported by a NYI grant.

\section{Peter Petersen}

Department of Mathematics

University of California at Los Angeles

Los Angeles, California 900242 


\section{Shunhui Zhu}

Department of Mathematics

Dartmouth College

Hanover, New Hampshire 03755

Received: September 11th, 1994; revised: November 23rd, 1994. 